\title{
Second order reductions of the WDVV Equations related to classical Lie algebras
}

\author{
L.K. Hoevenaars $\quad$ R. Martini
}

\begin{abstract}
We construct second order reductions of the generalized Witten-DijkgraafVerlinde-Verlinde system based on simple Lie algebras. We discuss to what extent some of the symmetries of the WDVV system are preserved by the reduction.
\end{abstract}

MSC Subj. Class. 2000: 35C05, $81 \mathrm{~T} 60$

Keywords: WDVV equations, prepotentials, Seiberg-Witten theory

\section{Introduction}

In two-dimensional topological conformal field theory the following remarkable system of third order nonlinear partial differential equations for a function $F$ of $N$ variables emerged [13, $[3]$

$$
F_{i} F_{1}^{-1} F_{j}=F_{j} F_{1}^{-1} F_{i} \quad i, j=1, \ldots, N
$$

Here $F_{i}$ is the matrix

$$
\left(F_{i}\right)_{k l}=\frac{\partial^{3} F}{\partial x_{i} \partial x_{k} \partial x_{l}} .
$$

Roughly speaking, this system expresses the conditions on a function whose third order derivatives form the structure constants of an associative, commutative algebra. Moreover, it is required that $F_{1}$ is a constant and invertible matrix. Usually this system is called the Witten-Dijkgraaf-Verlinde-Verlinde (WDVV) equations. Generalizations, not requiring $F_{1}$ to be constant, have been introduced and studied in the context of four- and five-dimensional $N=2$ supersymmetric gauge theory (see e.g. 6, 7, 9].

Although extremely difficult to solve in general, this overdetermined system (11) of nonlinear partial differential equations admits exact solutions. For instance, within the theory of Frobenius manifolds, a substantial class of polynomial solutions has been constructed by Dubrovin 4] for any Coxeter group. Furthermore, for any gauge group, perturbative approximations to exact prepotentials in four-dimensional Seiberg-Witten theory satisfy the (generalized) WDVV equations. These solutions are of logarithmic type and may be constructed for any root system (see [6] and [8]). The corresponding five-dimensional theories give rise to trigonometric solutions, see e.g. 11, 9.

Inspired by the paper [1] we construct second order reductions of the (third order) WDVV system, related to the logarithmic solutions associated with classical Lie algebras. In [1, the authors' main result is a form of the WDVV equations resembling 
a cocycle condition

$$
\sum_{i, k} \frac{\partial T_{a i}}{\partial T_{b j}} \frac{\partial T_{b k}}{\partial T_{c i}} \frac{\partial T_{c l}}{\partial T_{a k}}=\delta_{l}^{j}
$$

where $T$ is the Hesse matrix of a function $F\left(x_{1}, \ldots, x_{N}\right)$. The main idea is to perform a change of variables from $x_{k}$ to a single row $T_{a}=\left\{T_{a k}\right\}$ of the Hesse matrix. All other elements of the Hesse matrix are functionally dependent on that row and the authors then show that the equations (2) are equivalent to the generalized WDVV system.

In fact, Braden and Marshakov go on to construct a second order reduction ${ }^{1}$ of the WDVV equations by studying the relations among the elements of the Hessian of the prepotential of type $A_{3}$. In this paper, we modify and largely extend the construction of such reductions to all Lie algebras of classical type in section 2 Finally, we discuss the effect of changes of the ultraviolet cut-off on the reductions in section 3

\section{Second order reductions for classical Lie alge- bras}

For any rank $N$ root system $R$ with positive roots $R^{+}$, we consider the following function

$$
\mathcal{F}\left(x_{1}, \ldots, x_{N}\right)=\frac{1}{2} \sum_{\alpha \in R^{+}}(\alpha, x)^{2} \ln \left(\frac{(\alpha, x)}{\Lambda}\right)
$$

where $x=\sum_{i} x_{i} v_{i}$ is a linear combination of basis vectors $v_{i}$ of the root space, and $(.,$.$) denotes the Euclidean inner product. Such functions are known as pure$ perturbative four-dimensional Seiberg-Witten prepotentials in the physics literature [12, 5], where they occur in the study of quantum Yang-Mills theories. The parameter $\Lambda$ occurs in the Seiberg-Witten theory as an energy cut-off scale, and is irrelevant for the WDVV equations since it has no effect on the third order derivatives of $\mathcal{F}$. The reductions we propose here are of second order, but as we will show in section 3 they turn out also to be independent of the value of $\Lambda$.

\subsection{The reduction for type $A$ prepotentials}

Naturally, the explicit form of the prepotential depends on the chosen basis $\left\{v_{i}\right\}$. Although this choice is usually irrelevant since linear transformations are symmetries of the WDVV equations, we will be dealing with second order reductions on which the choice may have an influence. The choice we make here leads to the well-known type $A$ Seiberg-Witten prepotential

$$
\mathcal{F}\left(x_{1}, \ldots, x_{N}\right)=\frac{1}{2} \sum_{i<j}\left(x_{i}-x_{j}\right)^{2} \ln \left(\frac{x_{i}-x_{j}}{\Lambda}\right)+\frac{1}{2} \sum_{i} x_{i}^{2} \ln \left(\frac{x_{i}}{\Lambda}\right)
$$

\footnotetext{
${ }^{1}$ A reduction of a system of differential equations for a single function $F$ is understood here to be a set of lower order equations for $F$ whose solutions are automatically solutions of the original system.
} 
For convenience we set $\Lambda=e^{\frac{3}{2}}$, after which the second order derivatives $T_{i j}$ of $\mathcal{F}$ are given by

$$
\begin{aligned}
e^{-T_{i j}} & =x_{i}-x_{j} \quad i<j \\
e^{T_{i i}} & =x_{i} \prod_{q>i}\left(x_{i}-x_{q}\right) \prod_{q<i}\left(x_{q}-x_{i}\right)
\end{aligned}
$$

These second order derivatives satisfy the following set of relations

$$
\begin{array}{ll}
e^{-T_{i k}}=e^{-T_{i j}}+e^{-T_{j k}} & \forall i<j<k \\
e^{-T_{i j}}=e^{\sum_{p} T_{i p}}-e^{\sum_{q} T_{j q}} & \forall i<j
\end{array}
$$

These relations are somewhat reminiscent of those occurring in Hirota's work on tau functions of integrable hierarchies [1], 14].

Starting from the relations (67), (77) one sees that the elements $T_{i j}$ of the Hesse matrix $T$ can be expressed in terms of a single row $T_{a}=\left\{T_{a k}\right\}$ for fixed $a$ as follows

$$
\begin{array}{ll}
T_{i j}=-\log \left(e^{-T_{i a}}+e^{-T_{a j}}\right) & i<a<j \\
T_{i j}=-\log \left(e^{-T_{a j}}-e^{-T_{a i}}\right) & a<i<j \\
T_{i j}=-\log \left(e^{-T_{i a}}-e^{-T_{j a}}\right) & i<j<a
\end{array}
$$

and

$$
\begin{array}{ll}
T_{i i}=\log \left(e^{-\sum_{p \neq i} T_{i p}}\left[e^{T_{i j}}+e^{\sum_{q} T_{a q}}\right]\right) & i<a \\
T_{i i}=\log \left(e^{-\sum_{p \neq i} T_{i p}}\left[-e^{T_{i j}}+e^{\sum_{q} T_{a q}}\right]\right) & i>a
\end{array}
$$

In general no relations exist between the $T_{a k}$ and they can be taken as independent.

Proposition 2.1. The relations (6), (7) form a second order reduction of the WDVV equations in the sense that any function $G\left(z_{1}, \ldots, z_{N}\right)$ whose second order derivatives $T_{i j}$ satisfy the reduction, automatically satisfies the WDVV equations.

Proof. Having a function $G(z)$ satisfying the reduction, we use the equations

$$
\begin{array}{rlrl}
e^{-T_{i j}} & =x_{i}-x_{j} & i<j \\
e^{T_{i i}} & =x_{i} \prod_{q>i}\left(x_{i}-x_{q}\right) \prod_{q<i}\left(x_{q}-x_{i}\right) &
\end{array}
$$

to introduce the objects $x_{i}(z)$. We already know from the study of the prepotential (44) that these equations are compatible with the reduction. Moreover it is easily seen that (10) can be used to define the $x_{i}$ uniquely. Since the objects $\frac{\partial T_{i j}}{x_{k}}$ are totally symmetric, the $T_{i j}$ can be integrated twice with respect to the $x_{i}$ (not the $z_{i}$ ) to obtain the known prepotential (4). We now have two functions, the well-known $\mathcal{F}(x)$ and the unknown $G(z)$, whose second order derivatives are equal $\frac{\partial^{2} \mathcal{F}}{\partial x_{i} \partial x_{j}}(x(z))=\frac{\partial^{2} G}{\partial z_{i} \partial z_{j}}(z)$. As shown in [10], due to this relation between the second order derivatives the unknown function $G(z)$ satisfies the WDVV equations since the prepotential $\mathcal{F}$ does. 


\begin{tabular}{|l|l|l|}
\hline$B_{N}$ & $\pm e_{i} \pm e_{j}, e_{i}$ & $\eta=\frac{1}{2}, \zeta=0$ \\
\hline$C_{N}$ & $\pm e_{i} \pm e_{j}, 2 e_{i}$ & $\eta=2, \zeta=2 \ln (2)$ \\
\hline$D_{N}$ & $\pm e_{i} \pm e_{j}$ & $\eta=\zeta=0$ \\
\hline
\end{tabular}

Table 1: The roots of the type $B, C, D$ Lie algebras together with the values of $\eta, \zeta$ occurring in the prepotential [11). Here $e_{i}$ denotes a vector with 1 in the $i$-th place and 0 elsewhere.

\subsection{The reduction for the remaining classical Lie algebras}

The roots in $\mathbb{R}^{N}$ of the remaining classical Lie algebras are given in table 1 Taking the standard Euclidean basis, the corresponding prepotentials are

$$
\begin{array}{r}
\mathcal{F}\left(x_{1}, \ldots, x_{N}\right)=\frac{1}{2} \sum_{i<j}\left(\left(x_{i}-x_{j}\right)^{2} \ln \left(\frac{x_{i}-x_{j}}{\Lambda}\right)+\left(x_{i}+x_{j}\right)^{2} \ln \left(\frac{x_{i}+x_{j}}{\Lambda}\right)\right) \\
+\eta \sum_{i} x_{i}^{2} \ln \left(\frac{x_{i}}{\Lambda}\right)+\zeta \sum_{i}\left(x_{i}\right)^{2}
\end{array}
$$

with $\eta, \zeta$ as in table 1 Chosing $\Lambda=e^{\frac{6(N-1+\eta)+4 \zeta}{4(N-1+\eta)}}$, one finds

$$
\begin{aligned}
e^{-T_{i j}} & =\frac{x_{i}-x_{j}}{x_{i}+x_{j}} \\
e^{T_{i i}} & =x_{i}^{2 \eta} \prod_{q>i}\left(x_{i}^{2}-x_{q}^{2}\right) \prod_{q<i}\left(x_{q}^{2}-x_{i}^{2}\right)
\end{aligned}
$$

From these values of the second order derivatives of the prepotential one can derive the following relations among them

$$
\begin{aligned}
\operatorname{coth}\left(\frac{T_{i k}}{2}\right) & =\operatorname{coth}\left(\frac{T_{i j}}{2}\right) \operatorname{coth}\left(\frac{T_{j k}}{2}\right) & & \forall i<j<k \\
e^{T_{i i}-T_{j j}} & =\left[\operatorname{coth}\left(\frac{T_{i j}}{2}\right)\right]^{2 \eta+N-2} \frac{\prod_{n \neq j} \sinh \left(T_{j n}\right)}{\prod_{m \neq i} \sinh \left(T_{i m}\right)} & & \forall i<j
\end{aligned}
$$

We therefore have the following result

Proposition 2.2. The relations (14), (15) form a second order reduction of the $W D V V$ equations in the sense that any function $G\left(z_{1}, \ldots, z_{N}\right)$ whose second order derivatives $T_{i j}$ satisfy the reduction, also satisfies the WDVV equations.

Proof. The proof follows the same lines as the proof of proposition 2.1

\section{The role of $\Lambda$}

The parameter $\Lambda$ plays the role of the energy cut-off in Seiberg-Witten theory. Changing this parameter amounts to adding a second order polynomial to the prepotential, which is clearly a symmetry of the third order WDVV equations. The reductions proposed in this paper are of second order, and so the value of $\Lambda$ can in principal make a difference. We will show however that this is not the case. 
Proposition 3.1. Adding a second order polynomial preserves the solution set of the reduction (6), (7) iff the polynomial is a multiple of $\left(\sum_{i<j}\left(x_{i}-x_{j}\right)^{2}+\sum_{i} x_{i}^{2}\right)$. Similarly, addition of a second order polynomial preserves the solution set of the reduction (14), (15) iff it is of the form $\sum_{i \neq j} n \pi \sqrt{-1} x_{i} x_{j}+\xi \sum_{i} x_{i}^{2}$ with arbitrary integer $n$ and arbitrary parameter $\xi$.

Proof. Adding a polynomial $\sum_{i, j} a_{i j} x_{i} x_{j}$ to a generic solution of the type $A_{N}$ reduction, one finds that (6) restricts $a_{i j}=c$ for $i \neq j$ and some common constant $c$. Then it is clear that (7) restricts $a_{i i}=-N c$. This leads to the assertion of the lemma regarding the type $A$ reduction. Similarly (14) restricts the polynomial to $\sum_{i<j} n \pi \sqrt{-1} x_{i} x_{j}+\sum_{i} a_{i i} x_{i}^{2}$, and (15) restricts the $a_{i i}$ further.

A change $\Lambda \rightarrow e^{-2 \xi} \Lambda$ in (4) leads to an addition of a term $\xi\left(\sum_{i<j}\left(x_{i}-x_{j}\right)^{2}+\sum_{i} x_{i}^{2}\right)$ which is a symmetry of the type $A$ reduction. Similarly, a change $\Lambda \rightarrow e^{-\frac{\xi}{N-1+\eta}} \Lambda$ in (11) gives an additional term $\xi \sum_{i} x_{i}^{2}$ which in turn is a symmetry of the $B C D$ reduction.

\section{Remarks}

The generalized WDVV equations are known to be invariant under the following two types of transformations [4, 2]: linear transformations of the coordinates and so-called duality transformations. The duality transformations comprise a group of contact symmetries acting as constant symplectic transformations on a vector containing the coordinates $x_{i}$ as one half of its indices and the first order derivatives $F_{i}$ of the prepotential as the other half. It is readily observed that the reductions (6), (7) and (14), (15) are not invariant under general linear coordinate changes nor general symplectic transformations. This implies among other things that the choice of basis in the root space is relevant for the reduction one obtains.

A purpose of having a reduction might be to see if it can be easily solved, thus gaining new solutions to the WDVV equations. As shown above, the solutions of the reductions consist of the functions $G(z)$ whose Hesse matrix equals that of the perturbative prepotentials $\mathcal{F}(x)$ for classical Lie algebras: $\frac{\partial^{2} G}{\partial z_{i} \partial z_{j}}(z)=\frac{\partial^{2} F}{\partial x_{i} \partial x_{j}}(x(z))$. Since this type of relation between two prepotentials was already studied in the literature (see e.g. [10]) the reduction does not yield new information in this sense.

\section{References}

[1] H. W. Braden and A. Marshakov. WDVV equations as functional relations. Phys. Lett. B, 541(3-4):376-383, 2002.

[2] B. de Wit and A. V. Marshakov. Electric-magnetic duality and the WittenDijkgraaf-Verlinde-Verlinde equation. Teoret. Mat. Fiz., 129(2):230-238, 2001.

[3] R. Dijkgraaf, H. Verlinde, and E. Verlinde. Topological strings in d ; 1 . Nucl. Phys., B352:59-86, 1991.

[4] B. Dubrovin. Geometry of 2D topological field theories. In Integrable systems and quantum groups (Montecatini Terme, 1993), pages 120-348. Springer, Berlin, 1996. 
[5] A. Klemm, W. Lerche, S. Yankielowicz, and S. Theisen. Simple singularities and $\mathrm{N}=2$ supersymmetric Yang-Mills theory. Phys. Lett., B344:169-175, 1995.

[6] A. Marshakov, A. Mironov, and A. Morozov. WDVV-like equations in N = 2 SUSY Yang-Mills theory. Phys. Lett., B389:43-52, 1996.

[7] A. Marshakov, A. Mironov, and A. Morozov. More evidence for the WDVV equations in $\mathrm{N}=2$ SUSY Yang- Mills theories. Int. J. Mod. Phys., A15:1157, 2000 .

[8] R. Martini and P. K. H. Gragert. Solutions of WDVV equations in SeibergWitten theory from root systems. J. Nonlinear Math. Phys., 6(1):1-4, 1999.

[9] R. Martini and L. K. Hoevenaars. Trigonometric solutions of the WDVV equations from root systems. Lett. Math. Phys., 65(1):15-18, 2003.

[10] A. Mironov and A. Morozov. Covariance of WDVV equations. Phys. Lett. B, 424(1-2):48-52, 1998.

[11] N. Nekrasov. Five-dimensional gauge theories and relativistic integrable systems. Nuclear Phys. B, 531(1-3):323-344, 1998.

[12] N. Seiberg and E. Witten. Electric - magnetic duality, monopole condensation, and confinement in $\mathrm{n}=2$ supersymmetric yang-mills theory. Nucl. Phys., B426:19-52, 1994.

[13] E. Witten. Two-dimensional gravity and intersection theory on moduli space. In Surveys in differential geometry (Cambridge, MA, 1990), pages 243-310. Lehigh Univ., Bethlehem, PA, 1991.

[14] A. Zabrodin. A survey of hirota's difference equations. solv-int/9704001. 